Trauma Berufskrankh 2018 20 (Suppl 3):S141-S149 https://doi.org/10.1007/s10039-018-0364-0 Online publiziert: 4. April 2018

(c) Springer Medizin Verlag GmbH, ein Teil von Springer Nature 2018

CrossMark

\author{
T. Pitzen' • B. Al Kahwagi' · G. Ostrowski' · T. Welk² · C. Pöckler-Schöninger ${ }^{2}$. \\ J. Drumm' - M. Ruf \\ 'ZWOT, Zentrum für Wirbelsäulenchirurgie, Orthopädie und Traumatologie, SRH-Klinikum Karlsbad \\ Langensteinbach, Karlsbad, Deutschland \\ ${ }^{2}$ Radiologie, SRH-Klinikum Karlsbad Langensteinbach, Karlsbad, Deutschland
}

\section{Akutversorgung und Spätfolgen bei kindlichen Verletzungen der Halswirbelsäule}

Nur 1-2\% aller kindlichen Traumen sind Halswirbelsäulentraumen, und nur 0,2\% aller kindlichen Brüche sind Wirbelbrüche [4]. Das Verhältnis von Jungen zu Mädchen beträgt dabei 2:1 [6,7]. Im Kindesalter überwiegen die zervikalen Verletzungen [7], in der Adoleszenz thorakolumbale Frakturen. Ursächlich dafür ist, dass bei Kleinkindern der Kopf im Vergleich zum Rumpf überproportional groß ist und eine hohe Flexibilität der Halswirbelsäule (HWS) auf eine zusätzlich noch schwach ausgebildete Muskulatur trifft. Entsprechend können Traumasituationen zu einer plötzlichen Überlastung im Bereich der HWS führen, und die HWS ist dagegen wenig geschützt. Dabei kann als Regel gelten: Je jünger das an der HWS verletzte Kind ist, desto kranialer liegt die Verletzung [5].

Die wesentlichsten Aspekte im Ablauf der Versorgung des an der HWS verletzten Kindes sind die frühzeitige Diagnose und dann die zügige, adäquate Versorgung. Dies ist die wahrscheinlich beste Ausgangssituation, um Spätfolgen zu vermeiden, denn diese würden das Kind ein Leben lang behindern.

Entsprechend behandelt dieser Beitrag die Akutversorgung und Spätfolgen bei kindlichen Verletzungen der Halswirbelsäule.

\section{Akutphase \\ Klinische und radiologische Diagnostik}

Klinisch ist zunächst eine sorgfältige traumabezogene und gezielte Anamnese zu erheben, die auch den Traumamechanismus einschließt. Neben der Bewertung des Unfallmechanismus wird dann eine sorgfältige klinische inklusive neurologische Untersuchung gefordert. Lokalbefunde, die man erheben sollte, sind Inspektion auf Verletzungszeichen, Verformungen und die Palpation des gesamten Rückens (Druck und Klopfschmerz, Stufen, Versetzungen, tastbare Lücken zwischen Dornfortsätzen, Hämatome, Kontusionen, Muskelhartspann). Vervollständigt wird die Basisuntersuchung mit der Prüfung der Funktionsfähigkeit, insbesondere der Rotationsfähigkeit im Bereich der HWS. Die orientierende neurologische Untersuchung der Sensibilität, der Motorik und der Reflexe ist selbstverständlich [9].

Röntgenuntersuchungen der Halswirbelsäule in 2 Ebenen und Denszielaufnahme sind nach wie vor unverzichtbar. $\mathrm{Zu}$ beurteilen sind Wirbelkörperhöhe, Alignement der gesamten HWS, Homogenität der lordotischen/ kyphotischen Schwingungen, Form- und Kontinuitätsveränderungen des Wirbelkörpers, die interspinöse Distanz, Stellung der Gelenke („on-over the top“), Stellung Dens mittig oder dezentriert, Stellung der Massa lateralis C1-2 und prävertebrale Weichteilschatten, Distanzierung und Stufenbildung der Dornfortsätze, Unterbrechung, Auswölbung und Erniedrigung des Wirbelkörpers,
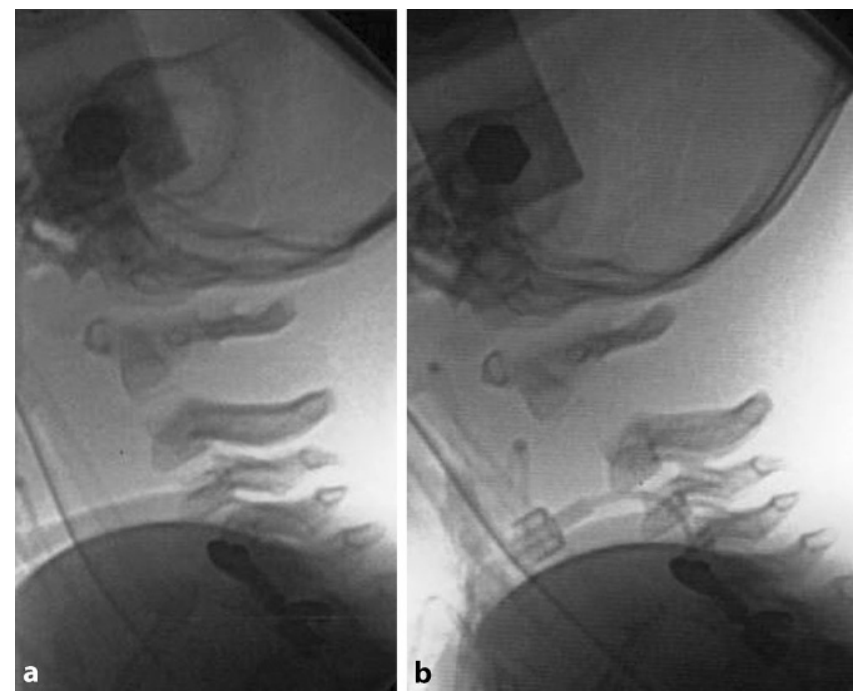

Abb. $1<$ a Seitliches Röntgenbild der Halswirbelsäule eines Kindes mit Densfraktur. b Hier zeigt sich eine hochgradige Instabilität bei Distraktion 


Übersichten
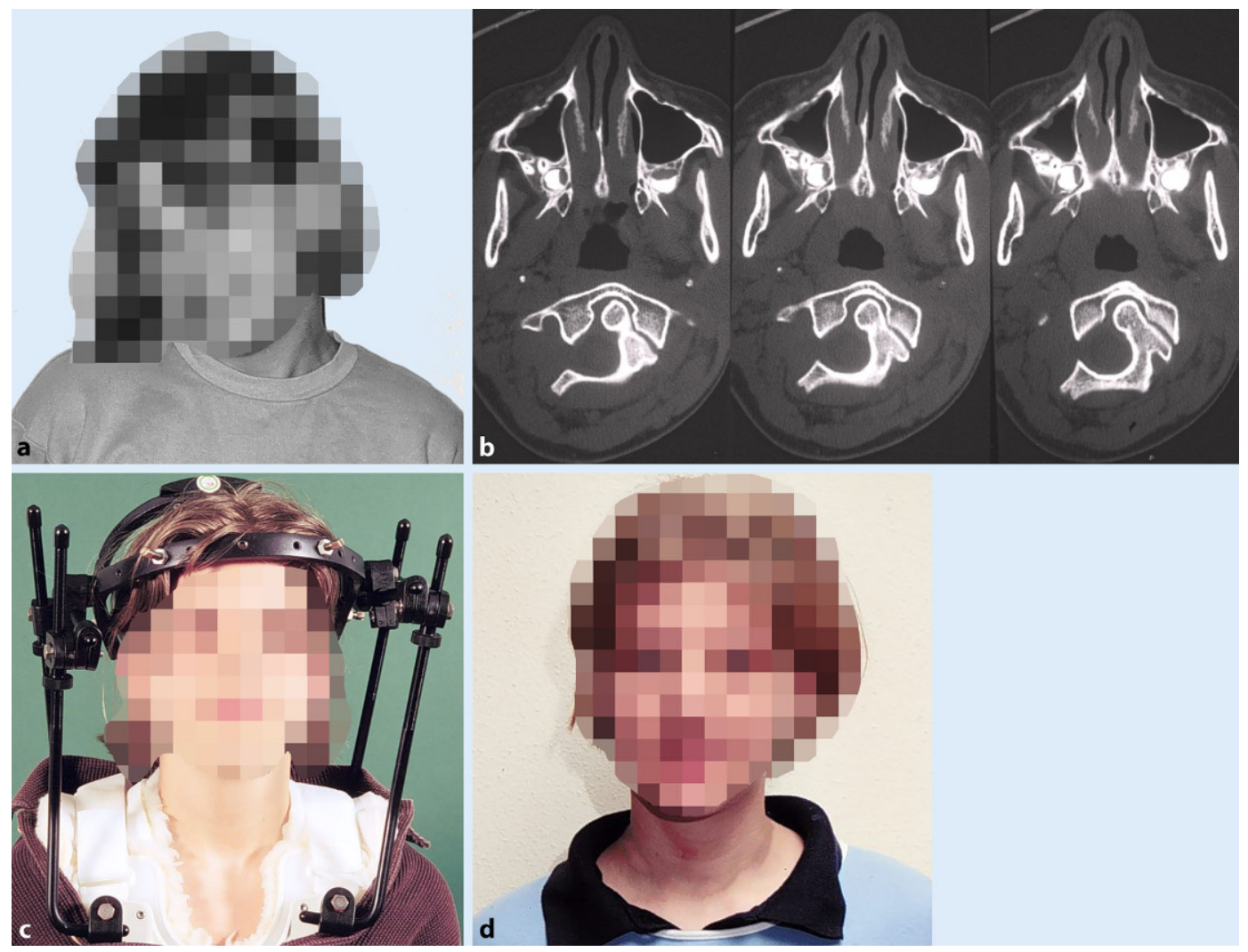

Abb. $2 \triangleleft$ a Klinisches Bild eines 11-jährigen Mädchens mit Rotationsfehlstellung nach einem Sturz von der Schaukel. b Die transversale Computertomographie zeigt eine Rotationsluxation C1/C2. c Patientin im Halo immobilisiert. d Klinisches Bild 6 Jahre nach der Abnahme des Halo-Body-Jackets
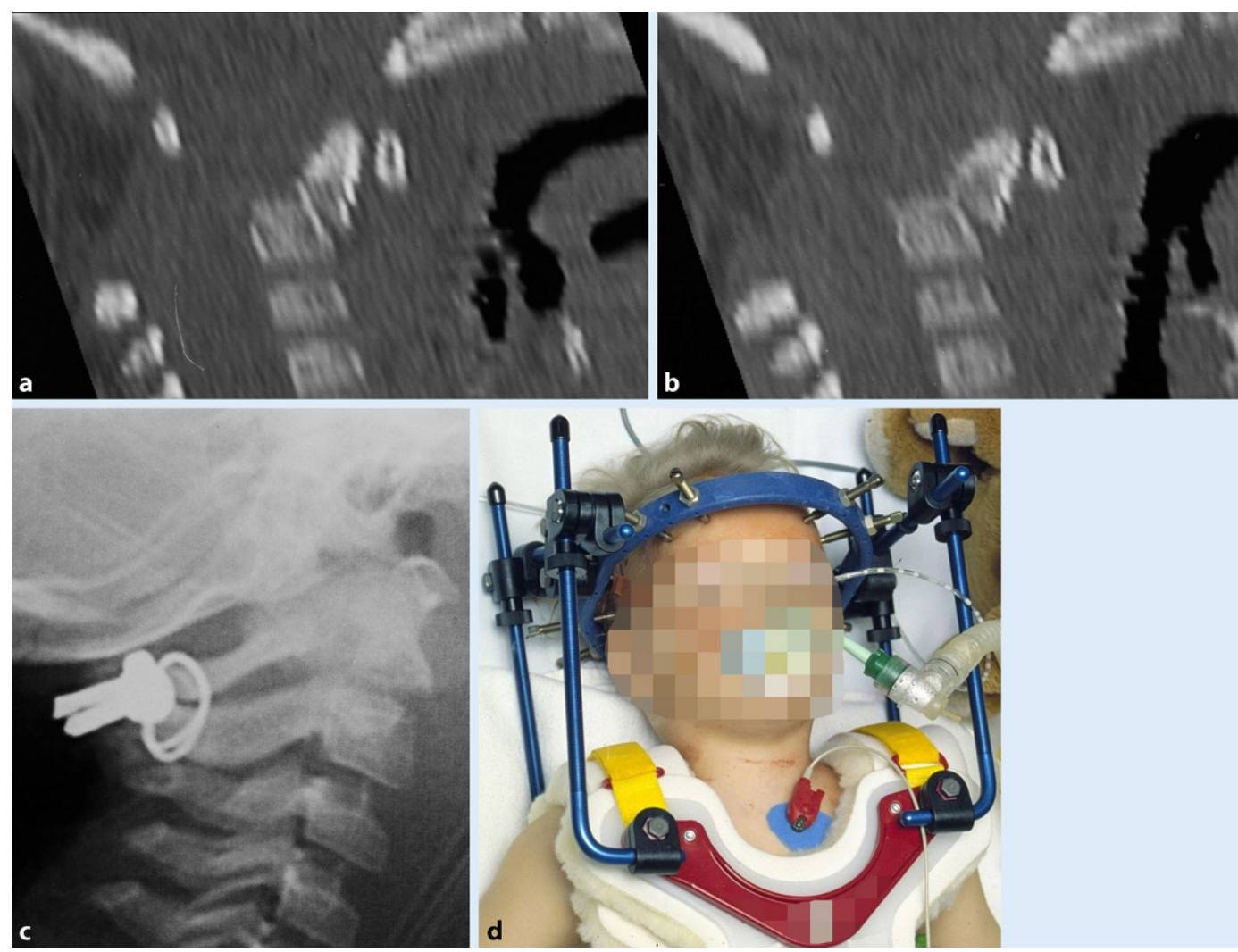

Abb. $3<$ a, b Sagittale Computertomographie(CT)-Rekonstruktion eines 1,5 Jahre alten Mädchens nach PKW-Unfall mit Densepiphyseolyse. c Seitliches Röntgenbild der Halswirbelsäule. d Klinisches Bild im Zustand nach Versorgung mit Drahtcerclage und Immobilisierung im Halo 
eine Translation zweier Hinterkanten und Größenänderungen des Neuroforamens. Alle diese Zeichen können auf eine Verletzung der HWS hinweisen.

Dabei muss beachtet werden, dass bei Kleinkindern jünger als 2 Jahre eine falsch positive kyphotische Fehlstellung aufgrund des vergrößerten Kopfes zu erwarten ist. $\mathrm{Zu}$ beachten ist außerdem, dass eine HWS-Röntgenaufnahme bei Kindern, die jünger als 6 Jahre alt sind, aufgrund der noch fehlenden Verknöcherung schwierig zu beurteilen ist. Beispiele hierfür sind: Der Knochenkern an der Densspitze erscheint im 6. Lebensmonat und verschmilzt um das 12. Lebensjahr mit dem Dens axis. Dies kann mit einer Fraktur verwechselt werden. Diagnostische Probleme können auch auftreten durch die noch offene Apophyse von C2, die physiologische Keilform der Wirbelkörper im Kindesalter und die bekannte Pseudoluxation C2-3 [16]. Bei Kindern ist der atlantodentale Abstand größer: beim Kind bis zum 7. Lebensjahr bis zu $5 \mathrm{~mm}$, beim Erwachsenen $3 \mathrm{~mm} \mathrm{[3].}$

Zur Beurteilung der knöchernen Struktur der HWS ist eine Computertomographie (CT) notwendig, insbesondere bei Verdacht auf Frakturen, Rotationsfehlstellungen und Luxationen der HWS. Diese sind nur mit der CT ausreichend darstellbar. Die CT ist auch unverzichtbar zur Planung einer operativen Therapie mit Implantaten, insbesondere auch, um die Lage der Arteria vertebralis in Relation zum Knochen darzustellen. Bei Polytraumen ist die CT (Traumaspirale) unverzichtbar, um einen schnellen Überblick sowohl über das Ausmaß der Wirbelsäulenverletzungen als auch evtl. Begleitverletzungen zu erhalten. Appliziert man Kontrastmittel, kann dann später auch noch eine Angiographiesequenz zur Darstellung der A. vertebralis gemacht werden.

Der notwendige Ausschluss diskoligamentärer Instabilitäten macht die Magnetresonanztomographie (MRT) bei den kindlichen Verletzungen zur wichtigsten Diagnostik, da die MRT eine sehr hohe Sensitivität hat und bei fehlendem Nachweis einer Verletzung in der Regel keine Funktionsaufnahmen mehr durchgeführt werden müssen [4]. Bei Vorliegen neurologischer Symptome ist die MRT Pflicht zur Beurteilung einer Bandscheibenpathologie, zum sicheren Ausschluss einer ligamentären Verletzung, zur Abschätzung eines Wirbelkörperödems [8] sowie einer Myelonkompression oder -verletzung [12]. Im Kindesalter kommt noch dazu, dass man durch die Anwendung der MRT Strahlen vermeidet. Nachteilig bei der MRT ist nach wie vor die Verfügbarkeit außerhalb der Kernarbeitszeit, v. a. nachts ist dies nicht in allen Kliniken möglich. Weiterhin ist zu bedenken, dass die MRT bei kleinen Kindern in Narkose durchgeführt werden muss. Der organisatorische Aufwand ist also erheblich.

Die Anwendung der CT vs. MRT bei Kindern ist vor diesem Hintergrund immer wieder eine wichtige Frage. Die Vorteile der MRT (s. oben) sind unbestreitbar, die Verfügbarkeit manchmal aber ein großes Problem. Andererseits gibt auch die CT wichtige Informationen (s. oben), bedeutet aber eine Strahlenbelastung.

\section{Versorgung}

Die therapeutische Versorgung des verletzten Kindes sollte zügig, aber dennoch sorgfältig geplant werden. Sie sollte so bald wie möglich in einem Zentrum mit geeigneter Ausrüstung und Erfahrung durchgeführt werden, wofür alle entsprechenden Orthesen, Verfahren und Implantate vorzuhalten sind. Auch wenn rasches Handeln wichtig ist, sollte dennoch nicht in Übereile gehandelt werden. So sollte auch z. B. nicht übereilt Traktion auf die HWS ausgeübt werden, denn diese Verletzungen sind oft hoch instabil, und Distraktion kann die Situation verschlimmern (• Abb. 1).

Anhand der im Folgenden dargestellten Fallbeispiele möchten wir zum einen die wichtigsten Formen der Verletzungen der HWS bei Kindern beschreiben und zum anderen auf mögliche Probleme in der Akutversorgung und der Spätphase hinweisen.

\section{C1-2-Rotationsdislokationen}

Der erste Fall ist der eines 11-jährigen Mädchens nach Sturz von der Schaukel (- Abb. 2a). Die Diagnose lautete „psychogene Schiefhaltung " bei schwieriger
Trauma Berufskrankh 2018 · 20 (Suppl 3): S141-S149

https://doi.org/10.1007/s10039-018-0364-0

(c) Springer Medizin Verlag GmbH, ein Teil

von Springer Nature 2018

T. Pitzen • B. Al Kahwagi • G. Ostrowski • T. Welk · C. Pöckler-Schöninger · J. Drumm . M. Ruf

Akutversorgung und Spätfolgen bei kindlichen Verletzungen der Halswirbelsäule

Zusammenfassung

Kindliche Verletzungen im Bereich der Halswirbelsäule sind selten. Wesentlich sind eine frühzeitige Diagnosestellung und die rasche, konsequente Therapie. Dies ist die wahrscheinlich beste Ausgangssituation, um Spätfolgen zu vermeiden, denn diese würden das Kind ein Leben lang behindern. Entsprechend behandelt dieser Beitrag die Akutversorgung und Spätfolgen bei kindlichen Verletzungen der Halswirbelsäule.

Schlüsselwörter

Fraktur - Dislokationen - Röntgen - Therapie . Kyphose

Acute treatment and delayed sequelae of pediatric injuries of the cervical spine

\section{Abstract}

Pediatric injuries in the region of the cervical spine are rare. Timely diagnostics and a rapid and consistent treatment are essential. This is probably the best initial situation to avoid delayed sequelae as these would mean lifelong impairment for the child. Therefore, this article deals with the acute treatment and delayed sequelae of pediatric injuries of the cervical spine.

Keywords

Fracture $\cdot$ Dislocation $\cdot$ Medical history taking $\cdot$ X-rays $\cdot$ Treatment $\cdot$ Kyphosis 

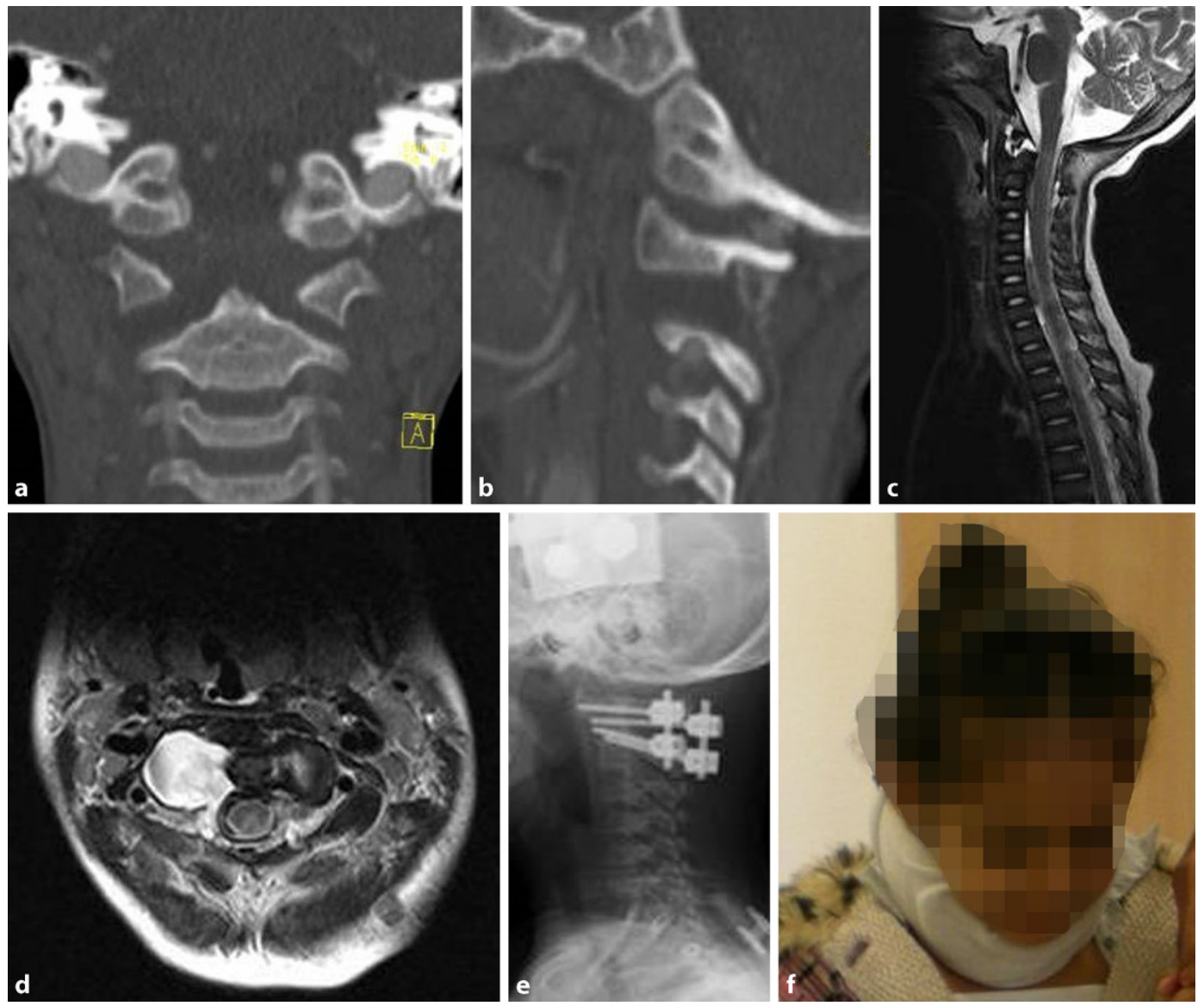

Abb. $4<$ a, b Sagittale und frontale Computertomographie(CT)-Rekonstruktion eines 3-jährigen Mädchens nach einem PKW-Unfall mit C1/C2-Dislokation. c, d Die Magnetresonanztomographie zeigt die Einblutung in das Facettengelenk sowie Liquoraustritt (Liquorzele) im sagittalen und axialen Schnitt.e, f Postoperatives seitliches Röntgenbild der Halswirbelsäule (e) und klinisches Bild 2 Jahre nach Versorgung ( $f$ )

sozialer Situation. Die CT zeigt, dass dies nicht zutrifft, es fand sich eine Rotationsluxation C1/C2 (• Abb. 2b). Die Versorgung erfolgte hier durch geschlossene Reposition in Narkose und Relaxation, dann schloss sich eine 3-monatige Immobilisation im Halo-Body-Jacket an (- Abb. 2c; [17]). Das klinische Ergebnis Jahre später zeigt - Abb. 2d. Es ist nach unseren Erfahrungen wesentlich, hier rasch zu handeln, denn je länger sich die Diagnose und entsprechend die Therapie verzögern, desto invasiver wird die Therapie [14]. Bei solchen Rotationsdislokationen im Bereich C1-2 ist es auch möglich, die Reposition in Narkose und Relaxation transoral durchzuführen. Dazu wird direkter Druck auf die nach ventral luxierte Massa lateralis ausgeübt, ggf. unter leichter Distraktion. Der Repositionserfolg sollte dann im Halo-Body-Jacket für ca. 6 bis 12 Wochen gesichert werden [17].

\section{Epiphyseolysen}

In manchen Fällen sind Läsionen aber so schwer oder instabil, dass bereits initial operiert werden muss, auch innerhalb des extrem wichtigen C1/2-Gelenkes. Dies zeigt der zweite klinische Fall: Ein 1-jähriges Mädchen erlitt im Rahmen eines PKW-Unfalls eine Densepiphyseolyse (•Abb. 3a, b). Das Mädchen wurde mit dorsaler Drahtcerclage C1/2 versorgt und zusätzlich im Halo immobilisiert (- Abb. 3c, d). Derklinische Verlauf war erfreulich.

\section{Ligamentäre C1/2-Zerreißungen}

Eine Operationsindikation bei schwerer ligamentärer Zerreißung ist auch in diesem Fall gegeben: Ein 3-jähriges Mädchens ist im Rahmen eines PKWUnfalls eine von 2 Überlebenden von 9 Insassen. Initial war sie tetraparetisch, hatte sich aber innerhalb weniger Tage dann gut erholt. Die MRT zeigt ein Auseinanderweichen der Gelenke $\mathrm{C} 1 / \mathrm{C} 2$, eine traumatische Duraläsion mit Liquoraustritt (Liquorzele) muss angenommen werden (• Abb. 4a-d). Die nächsten Bilder (• Abb. 4e, f) zeigen die postoperative Versorgung, und der klinische Verlauf bestätigte eine völlige Rückbildung der neurologischen Defizite.

\section{Atlasfrakturen}

Atlasfrakturen, wie hier in $\mathbf{A b b}$. $5 \mathrm{a}$ bei einem 12-jährigen Mädchen nach PKWUnfall zu sehen, sollten ebenfalls konsequent behandelt werden. Die gezeigte Fraktur gilt als instabil bei $7-8 \mathrm{~mm} \mathrm{Ge}$ lenküberhang. Dies drückt in der Regel den Verlust der Integrität des Atlasbogens aus. Letztendlich bedeutet dieses Auseinanderweichen, dass das Lig. transversum aus seiner Verankerung gerissen ist (- Abb. 5b). Entsprechend ist die Versorgung mit einer Orthese nicht sinnvoll [2]. Erst der Halo ermöglicht eine Distraktion und damit auch ein Zusammenrücken der Gelenke, wie in Vergleich • Abb. 5b, c zu sehen. Die - Abb. 5d-g zeigt die Patientin 2 Jahre nach Halo-Abnahme. Man kann diskutieren, ob man in einem sol- 

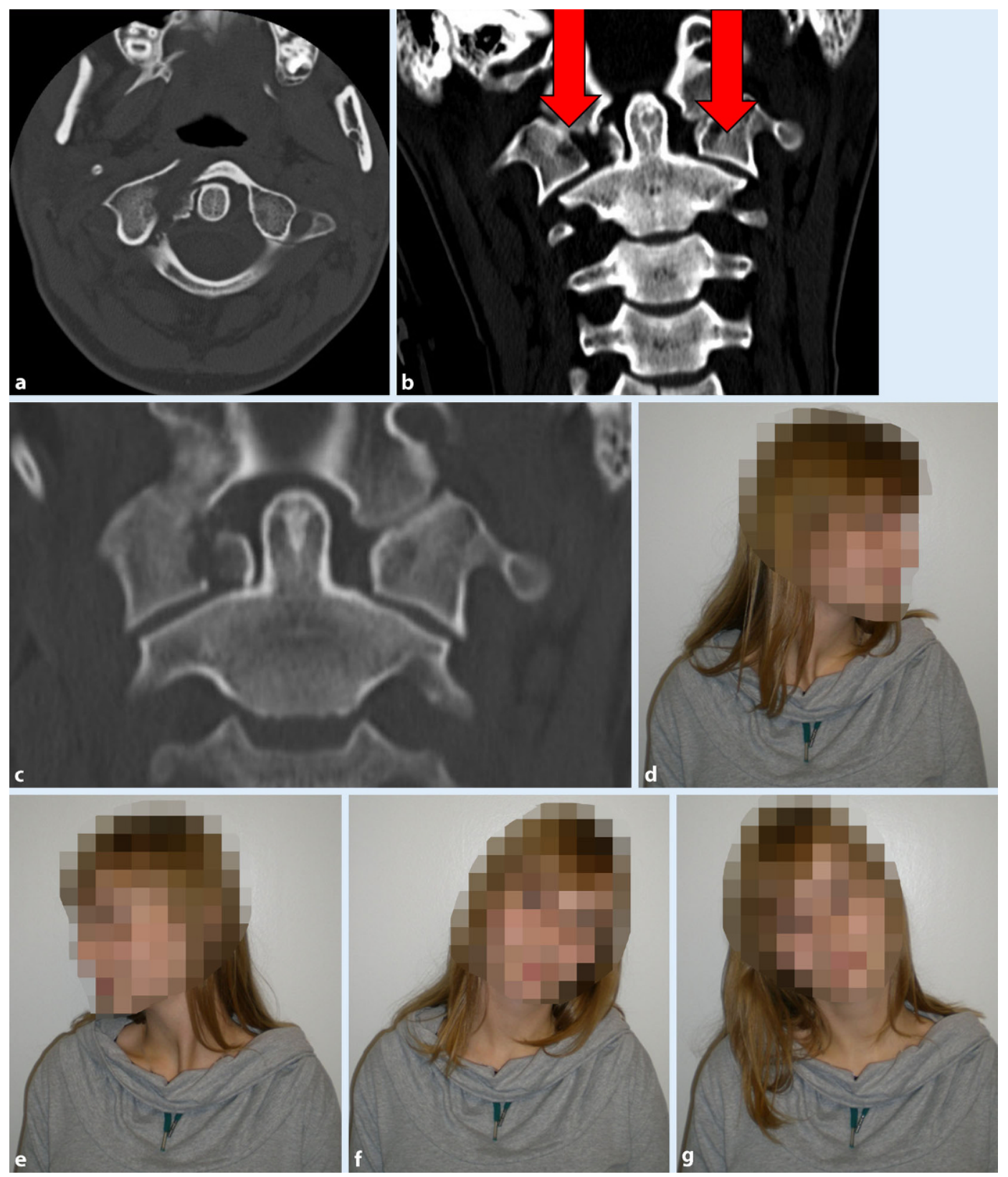

Abb. $5<$ a Transversale Computertomographie (CT) und $\mathbf{b}$ sagittale CT-Rekonstruktion eines 12 -jährigen Mädchens mit Atlasbogenfraktur (Jefferson-Fraktur). Die frontale CT-Rekonstruktion (b) ist in der Philadelphia-Orthese (c) mit Halo in 1 Monat später die Reposition ist deutlich erkennbar! d-g Patientin 2 Jahre nach Halo-Abnahme 

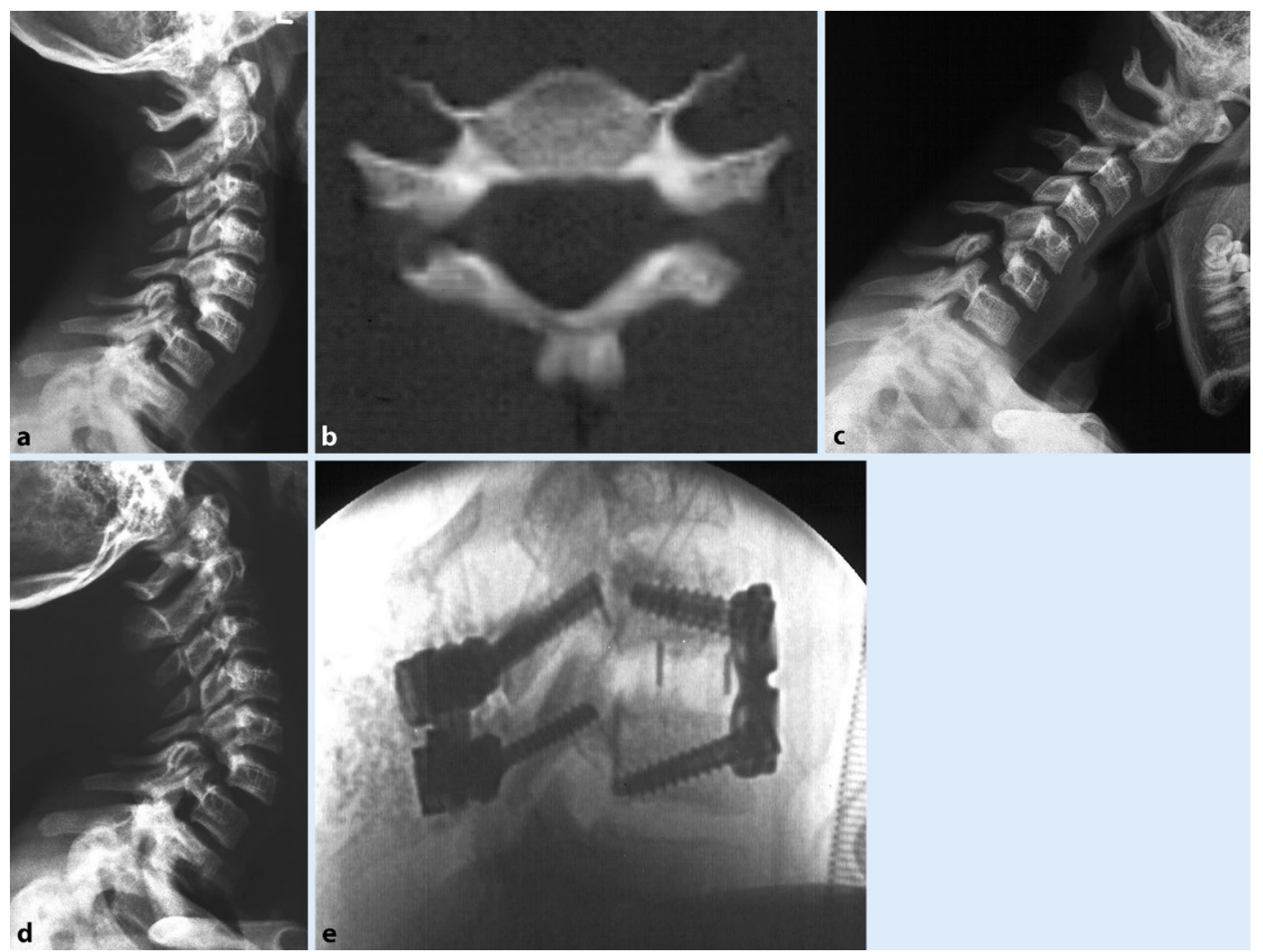

Abb. $6<$ a Seitliches Röntgenbild der Halswirbelsäule (HWS) eines 13-jährigen Jungen mit einer Läsion C6/C7. b Transversale Computertomographie, Lyse der Gelenke beidseitig. c, d Funktionsaufnahme der HWS: seitliche Reklination/Inklination. e Die intraoperative seitliche Durchleuchtung der HWS zeigt das Instrumentationsmaterial nach Versorgung dorsoventral
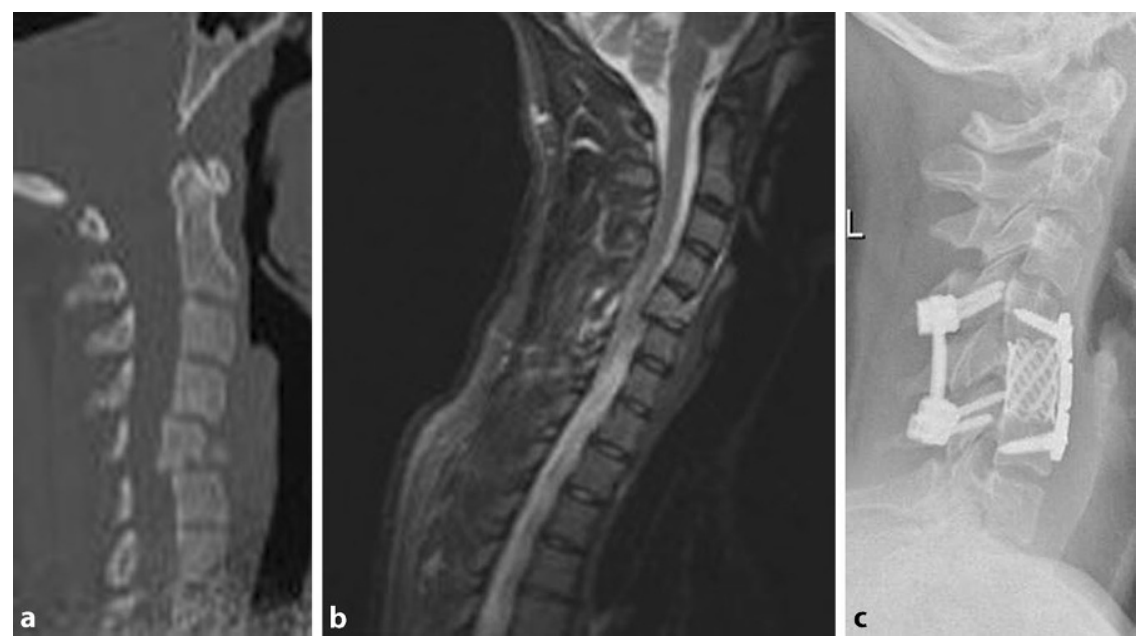

Abb. 7 A a Sagittale Computertomographie(CT)-Rekonstruktion und b sagittaler Magnetresonanztomographie(MRT)-Schnitt der Halswirbelsäule (HWS) eines 14 Jahre alten Mädchens nach PKW-Unfall als Beifahrerin mit Halswirbelkörper(HWK)-5-Berstungsfraktur. c Seitliches Röntgenbild der HWS als Verlaufskontrolle nach dorsoventraler Versorgung und Korporektomie C5. Kyphose oberhalb der korrekt lordotisch durchgeführten Versorgung

chen Fall auch eine Instrumentation anlegt, bei der eine Schraube in jede Massa lateralis des Atlas eingebracht wird und dann über einen Stab die beiden Schrauben verbunden werden, wobei man dann noch beide Seiten gegeneinander komprimieren kann [10]. In diesem Fall ha- ben wir dies nicht getan, weil die Fraktur bereits 4 Wochen alt war $[13,15]$.

\section{Subaxiale Verletzungen}

Im Fall eines 13-jährigen Ringers, der seit Jahren schon diesen Sport ausgeübt hat, sehen wir möglicherweise eine Ermüdungsfraktur C6/C7 (• Abb. 6a, b).
Diese ist in Funktion nicht zunehmend (-Abb. 6c, d) und in Narkose sofort reponierbar. Die Bilder ( $\bullet$ Abb. 6e) zeigen die Versorgung dorsoventral. Die Versorgung ist so stabil, dass nach Ausheilung nichts gegen die Wiederaufnahme des Sports sprach.

\section{Spätfolgen nach kindlichen Traumen}

Unter den Spätfolgen ist die Kyphose zu erwähnen. Die - Abb. 7 zeigt die Berstungsfraktur von Halswirbelkörper (HWK) 5 bei einem 14-jährigen Mädchen, versorgt mit einer ventrodorsalen Instrumentation. Die Kyphose oberhalb der korrekt lordotisch durchgeführten Versorgung ist zu erkennen (• Abb. 7c). Klinisch ist die junge Frau in einem perfekten Allgemeinzustand, die Kyphose muss aber beobachtet werden, um die Entwicklung einer Myelopathie nicht zu übersehen.

Leider gibt es auch immer wieder Kinder, die para- oder tetraplegisch oder -paretisch werden und bleiben oder sterben. In diesem Fall eines 8-jährigen Jungen mit einer $\mathrm{C} 1 / \mathrm{C} 2$-Luxation kam es nicht 

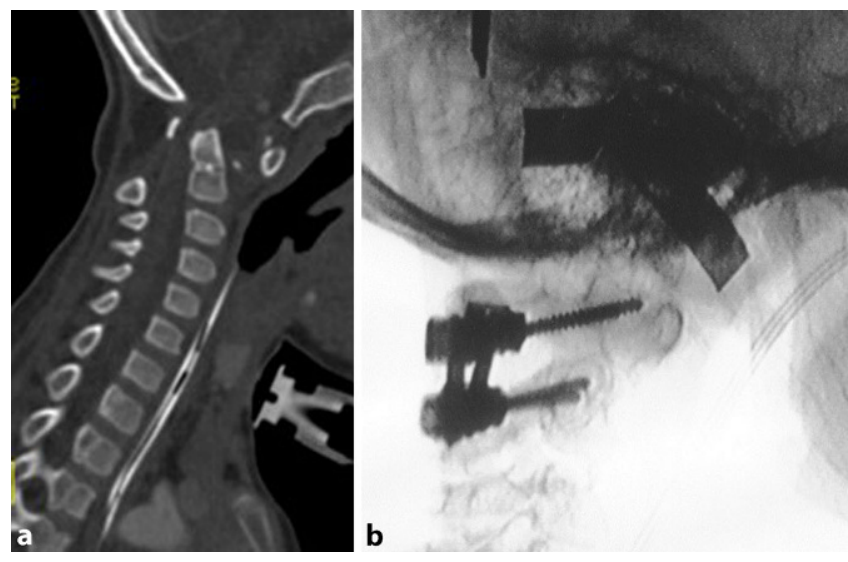

Abb. $8<$ a Sa-

gittale Com-

putertomogra-

phie(CT)-Rekonstruktion

der Halswirbelsäule

(HWS) eines 8-jähri-

gen Jungen mit ei-

ner C1/C2-Luxation.

b Die intraoperative

seitliche Durch-

leuchtung der HWS

zeigt die Instru-

mentation nach

Reposition
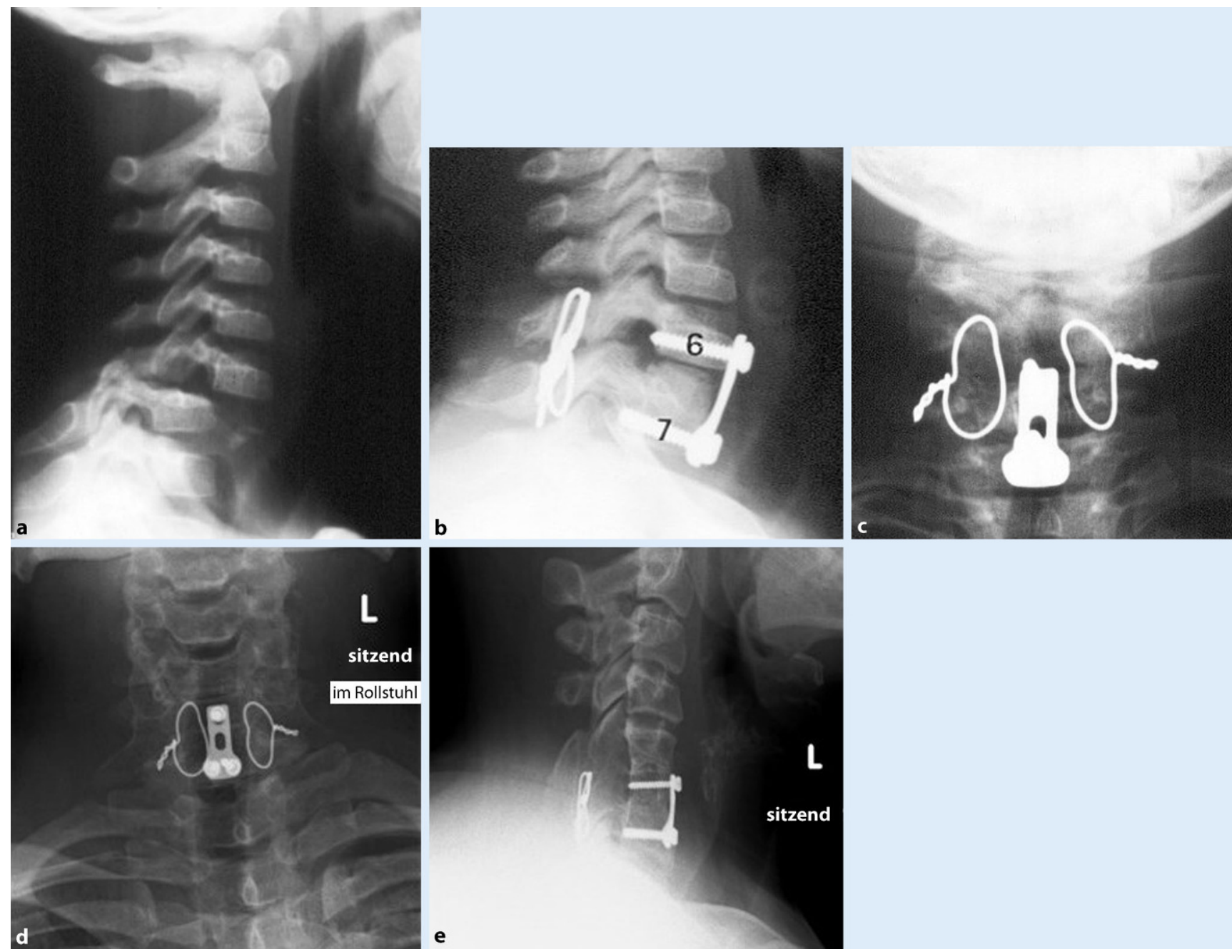

Abb. 9 \ a Seitliches Röntgenbild der Halswirbelsäule (HWS) eines 4-jährigen Jungen mit Luxationsfraktur C6/C7. b, c Postoperative Röntgenkontrolle derHWS in 2 Ebenen: reponierte Fraktur, Instrumentation. d, e Röntgenbilder der HWS in 2 Ebenen im Alter von 22 Jahren 


Übersichten
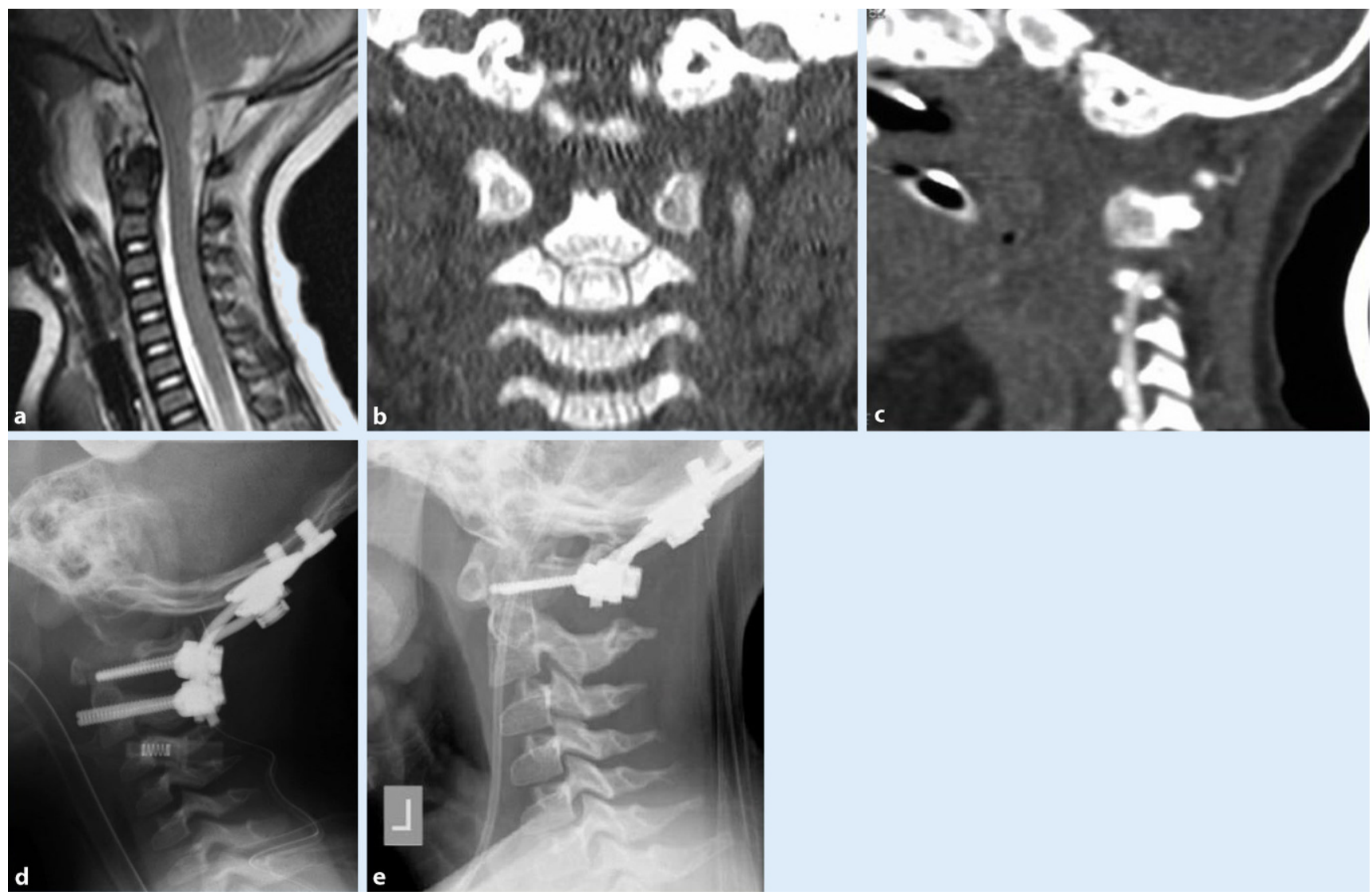

Abb. $10 \Delta$ a Sagittaler Magnetresonanztomographie(MRT)-Schnitt der Halswirbelsäule (HWS) eines 1,5 Jahre alten Mädchens nach PKW-Unfall mit atlantookzipitaler Luxation und höchstgradiger Tetraparese. b, c Sagittale und frontale AngioComputertomographie(CT)-Rekonstruktion der HWS. d Postoperatives seitliches Röntgenbild der HWS nach offener Reposition und C0-C2-Fusion. e Seitliches Röntgenbild der HWS nach Freigabe der Instrumentation auf C2

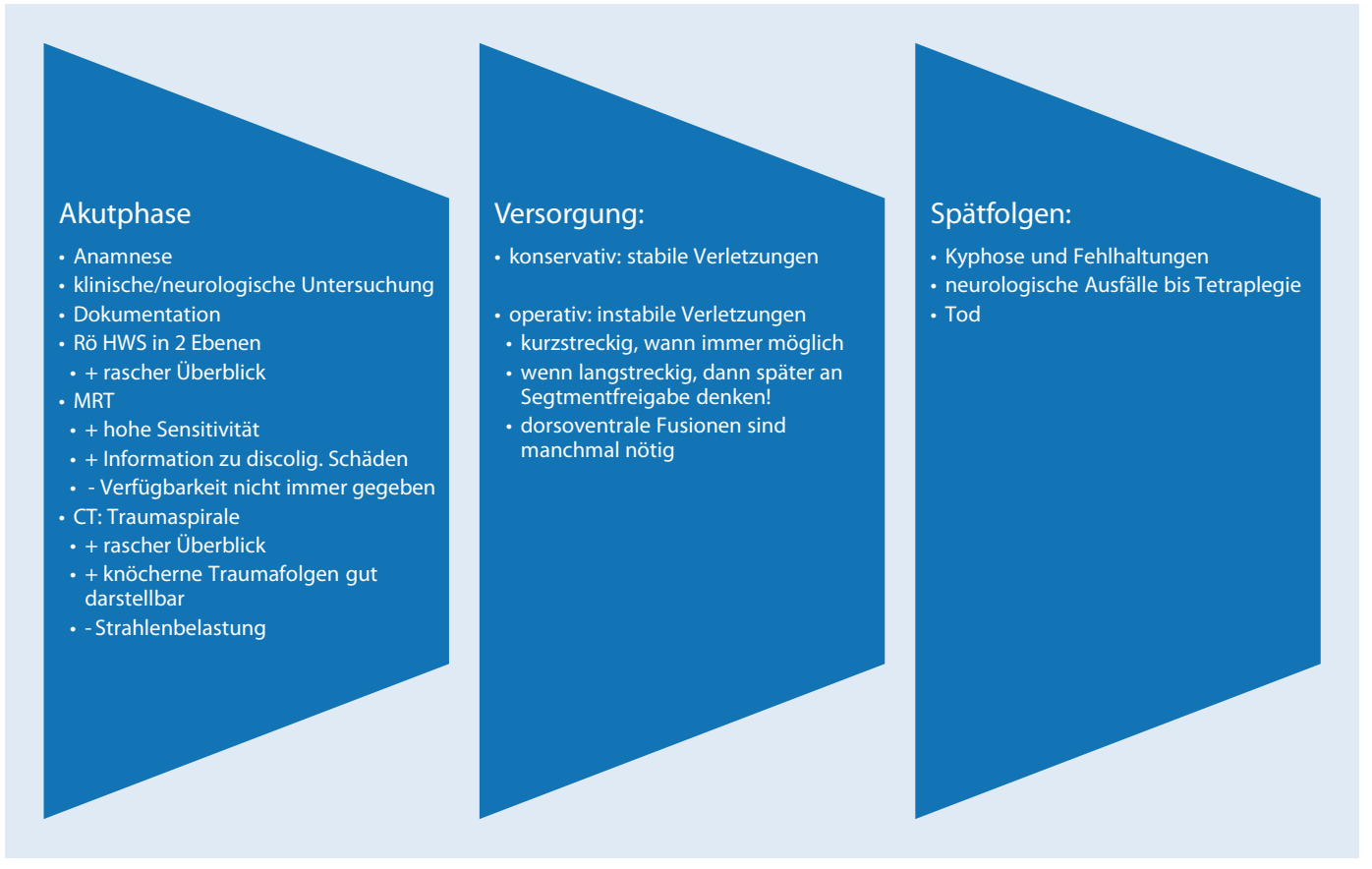

Abb. $11<$ Akutversorgung und Spätfolgen bei kindlichen Halswirbelsäulentraumen. + Vorteil - Nachteil, Rö HWS Röntgen der Halswirbelsäule, MRT Magnetresonanztomographie, discolig. diskoligamentäre Schäden, $C T$ Computertomographie 
zu einer Erholung trotz korrekter Versorgung (s. intraoperatives Bild [• Abb. 8]).

Ein weiterer Fall: Ein 4-jähriger Junge mit Luxationsfraktur $\mathrm{C} 6 / \mathrm{C} 7$ erlitt eine komplette Paraplegie unterhalb C7, die sich trotz perfekter Versorgung auch im Alter von 22 Jahren nicht gebessert hatte (• Abb. 9).

Ein 1,5 Jahre altes Mädchens mit atlantookzipitaler Luxation wurde initial im Halo-Body-Jacket behandelt, dann offen reponiert und C0 auf C2 versorgt. Nach 10 Monaten wurde die Instrumentation auf C2 freigegeben, an der Querschnittlähmung hat sich leider nichts geändert (- Abb. 10), aber die Patientin wird von der Segmentfreigabe (• Abb. 10e) profitieren.

Auch Todesfälle sind nach kindlichen Verletzungen der HWS beschrieben. Zu beachten ist, dass die Mortalität bei HWSTraumen bei Kindern höher ist als bei Erwachsenen $[1,11]$. Abb. 11 zeigt einen Überblick über die HWS Verletzungen im Kindesalter.

\section{Fazit für die Praxis}

- Kindliche Verletzungen der HWS sind selten. Das sorgfältige Erheben der Anamnese (inklusive des Unfallmechanismus), eine sorgfältige klinische inklusive neurologische Untersuchung und ausreichende Dokumentation sind erforderlich.

- Diagnostische bildgebende Verfahren sind die Röntgenuntersuchung der HWS in 2 Ebenen, ggf. Funktionsaufnahme oder Distraktionsaufnahme unter Durchleuchtung in Narkose und die MRT.

- In der Akutphase muss rasch und zügig, aber keinesfalls übereilt gehandelt werden, es muss zügig dokumentiert und die Versorgung geplant werden.

- Besonders im Kindesalter ist es sinnvoll, möglichst funktionserhaltend zu operieren, ggf. temporär zu instrumentieren, um später wichtige Segmente freigeben zu können. Fusionen sollten gemacht werden, wenn es nötig ist.

- Eine dauerhafte Tetraplegie ist möglich, aber eher selten.
- Kyphosen nach HWS-Verletzung sind bei Kindern nicht selten, aber oft klinisch nicht relevant. Um zu erkennen, ob sie relevant werden, ist ein enges klinisches Monitoring erforderlich.

- Komplikationen sind in der Akutphase unter anderem Todesfälle, die definitiv häufiger sind als in einem vergleichbaren Erwachsenenkollektiv.

\section{Korrespondenzadresse}

\section{Dr. B. Al Kahwagi}

ZWOT, Zentrum für Wirbelsäulenchirurgie, Orthopädie und Traumatologie, SRH-Klinikum Karlsbad Langensteinbach Guttmannstr. 1, 76307 Karlsbad, Deutschland Al-Kahwagi.Baraa@kkl.srh.de

\section{Einhaltung ethischer Richtlinien}

Interessenkonflikt. T. Pitzen, B. Al Kahwagi, G. Ostrowski, T. Welk, C. Pöckler-Schöninger, J. Drumm und M. Ruf geben an, dass kein Interessenkonflikt besteht.

Dieser Beitrag beinhaltet keine von den Autoren durchgeführten Studien an Menschen oder Tieren. Alle Patienten, die über Bildmaterial oder anderweitige Angaben innerhalb des Manuskripts zu identifizieren sind, haben hierzu ihre schriftliche Einwilligung gege ben. Im Falle von nicht mündigen Patienten liegt die Einwilligung eines Erziehungsberechtigten oder des gesetzlich bestellten Betreuers vor.

The supplement containing this article is not sponsored by industry.

\section{Literatur}

1. Brown RL, Brunn MA, Garcia VF (2001) Cervical spine injuries in children: a review of 103 patients treated consecutively at a level 1 pediatric trauma center.J Pediatr Surg 36(8):1107-1114

2. Böhm H, Kayser R, El Saghir H, Heyde CE (2006) Direct osteosynthesis of instable Gehweiler Type III atlas fractures. Presentation of a dorsoventral osteosynthesis of instable atlas frac tures while maintaining function. Unfallchirurg 109(9):754-760

3. Cattel HS, Fitzer DL (1965) Pseudosubluxation and other normal variations in the cervical spine in children. A studyofonehundred and sixty children. JBone Joint Surg Am 47:1295-1309

4. Garlepp J, Schnabel M, Fröhlich JJ, Gotzen L (1998) Ergebnisse der MRT - Diagnostik nach isolierter HWS-Distorsion. 62. Jahrestagung der Deutschen Unfallchirurgischen Gesellschaft für Unfallchirurgie e. V, S 102-103

5. Hill SA, Miller CA, Kosnik EJ et al (1984) Pediatric neck injuries. A clinical study. J Neurosurg 60:700-706
6. Jagannathan J, Dumont AS, Prevedello DM, Shaffrey Cl, Jane JA Jr (2006) Cervical spine injuries in pediatric athletes: mechanisms and management. Neurosurg Focus 21(4):E6

7. Jarvers J, Völker A, von der Höh N, Josten C, Heyde $C$ (2017) Verletzungen der Halswirbelsäule im Kindes- und Jugendalter. Wirbelsäule 1(3):171-182. https://doi.org/10.1055/s-0043-109437

8. Krakenes J, Kaale BR (2006) Magnetic resonance imaging assessment of craniovertebral ligaments and membranes after whiplash trauma. Spine $31: 2820-2826$

9. Krüger A (2012) Frakturen und Luxationen der Wirbelsäule. In: Ruchholtz S, Wirtz D (Hrsg) Orthopädie und Unfallchirurgie essentials, 2. Aufl. Thieme, Stuttgart https://doi.org/10.1055/b-00235715

10. Mikawa Y, Watanabe R, Yamano Y et al (1987) Fracture through a synchondrosis of the anterior arch of the atlas. J Bone Joint Surg Br 69:483

11. Eleraky MA, Theodore N, Adams M, Rekate HL, Sonntag VKH (2000) Pediatric cervical spine injuries: report of 102 cases and review of the literature. J Neurosurg Spine 92(1):12-17

12. Josten C, Heyde CE, Jarvers J-S (2016) Untersuchung der Halswirbelsäule. Orthop Unfallchir Up2date 11(1):43-62. https://doi.org/10.1055/s0041-106346

13. Pitzen T, Lane $C$, Goertzen D, Dvorak M, Fisher C, Barbier D, Steudel WI, Oxland T (2003) Anterior cervical plate fixation: biomechanical effectiveness as a function of posterior element injury. J Neurosurg 99(1 Suppl):84-90

14. Ruf M, Pitzen T, Drumm J (2017) Atlantoaxial rotatory dislocation: delayed diagnose will result in more invasive treatment options. Patient Saf Surg 11:5.https://doi.org/10.1186/s13037-016-0116-7

15. Ruf M, Melcher R, Harms J (2004) Transoral reduction and osteosynthesis $\mathrm{C} 1$ as a functionpreserving option in the treatment of unstable Jefferson fractures. Spine 29(7):823-827. https:// doi.org/10.1097/01.BRS.0000116984.42466.7E

16. Swischuk LE (1977) Anterior displacement of C2 in children: physiologic or pathologic? Pediatr Radiol 122:759-763

17. Weisskopf M, Naeve D, Ruf M, Harms J, Jeszenszky D (2005) Therapeutic options and results following fixed atlantoaxial rotatory dislocations. Eur Spine J $14(1): 61-68$ 